BULL. AUSTRAL. MATH. SOC.

VOL. $31(1985), 215-233$.

\title{
ABSOLUTE VALUES IN ORTHOGONALLY DECOMPOSABLE SPACES
}

\author{
SAdAYUKi Yamamuro
}

In an ordered Banach space which is orthogonally decomposable, we define the absolute value and its general properties are given. The results are used to study the properties of linear operators which satisfy Kato's inequality and the locality condition.

\section{Introduction}

Let $B$ be a real Banach space ordered by a closed and proper positive cone $B_{+}$. The (canonical) half-norm $N$ of $B$ has been defined in [2] by

$$
N(a)=\inf \left\{\|a+x\|: x \in B_{+}\right\} \text {for every } a \in B \text {. }
$$

When $B$ is a Banach lattice or the hermitian part of a $C^{*}$-algebra, we have $N(a)=\left\|a^{+}\right\|$and $N(-a)=\left\|a^{-}\right\|$, where $a^{+}$and $a^{-}$are the positive and the negative parts of an element $a$.

Generally, an ordered Banach space $B$ is said to be orthogonally decomposable if every element $a \in B$ is expressed in a form $a=b-c$ for some $b \in B_{+}$and $c \in B_{+}$such that $N(a)=\|b\|$ and $N(-a)=\|c\|$. It has been shown in [11], (3.4), that $B$ is orthogonally decomposable if and only if every $a \in B$ is expressed in a form $a=b-c$ for some $b \in B_{+}$and $c \in B_{+}$which are orthogonal in the following sense: there exist $b^{*} \in F(b) \cap B_{+}^{*}$ and $c^{*} \in F(c) \cap B_{+}^{*}$ such that

Received 28 September 1984.

(opyright Clearance Centre, Inc. Serial-fee code: 0004-9727/85 $\$ A 2.00+0.00$. 
$\left(b^{*}, c\right)=\left(b, c^{*}\right)=0$. Here, $F$ denotes the duality map of $B$ and $B_{+}^{*}$ is the dual cone in the dual $B^{*}$ of $B$, that is,

$$
F(b)=\left\{f \in B^{*}:(f, b)=\|b\|^{2}=\|f\|^{2}\right\},
$$

and

$$
B_{+}^{*}=\left\{f \in B^{*}:(f, x) \geq 0 \text { for all } x \in B_{+}\right\} \text {. }
$$

Let $B$ be an orthogonally decomposable space. Then, for every $a \in B$, the positive part $a_{+}$of $a$ is the set of all $b \in B_{+}$such that $a=b-c$ is an orthogonal decomposition for some $c \in B_{+}$. The negative part $a_{-}$is defined by $(-a)_{+}$. For a detailed discussion on these sets, we refer to $[11]$.

When $B$ is a Hilbert space $H$ ordered by a self-dual cone $H_{+}$, it is uniquely orthogonally decomposable, that is, every $\xi \in H$ has a unique decomposition $\xi=\xi_{+}-\xi_{-}$such that $\xi_{+} \in H_{+}, . \xi_{-} \in H_{+}$and $\left(\xi_{+}, \xi_{-}\right)=0$.

In general ordered Banach spaces $B$, an important rôle is played by a set-valued map $H$ defined by

$$
H(a)=\left\{f \in B_{+}^{*}:(f, a)=N(a)^{2}=\|f\|^{2}\right\} .
$$

It has been proved in [11], (1.7), that, if $b \geq a$ and $N(a)=\|b\|$,

$$
H(a)=F(b) \cap B_{+}^{*} \cap(b-a)^{0},
$$

where $x^{0}=\left\{f \in B^{*}:(f, x)=0\right\}$ for $x \in B$.

\section{Absolute values}

We shall always assume that $B$ is an orthogonally decomposable space. For $a \in B$ and $b \in B$, we have defined in [11] the set $a \vee b$ by $a \vee b=a+(a-b)_{-}$. Then the absolute value $\alpha(a)$ of $a \in B$ is the set defined by $\alpha(\alpha)=a \vee(-a)$.

(2.1). $x \in \alpha(a)$ if and only if $a=\frac{1}{2}(x+a)-\frac{1}{2}(x-a)$ is an orthogonal decomposition of $a$.

The proof follows immediately from the definition of the absolute value. It follows from (2.1) that $\alpha(a)$ is a closed and convex subset of $B_{+}$, and $\alpha(a)=\{a\}$ if $a \in B_{+}$. Furthermore, if $a=b-c$ is an 
orthogonal decomposition, we have $b+c \in \alpha(a)$. It is obvious that $\alpha(a) \subset a_{+}+a_{-}$.

When $B$ is uniquely orthogonally decomposable, $\alpha(a)$ consists of a single point $b+c$ for the unique orthogonal decomposition $a=b-c$. When $B$ is either a Banach lattice or the hermitian part of a $C^{*}$-algebra, $\alpha(a)$ may contain elements different from $|a|$, although $|\alpha|$ is the smallest element of $\alpha(\alpha)$ in the former case.

To consider the relations between the absolute values in $B$ and $B^{*}$, we need to restrict the size of $\alpha(\alpha)$. We shall denote by $\alpha_{c}(a)$ a subset of $\alpha(a)$ defined by

$$
\alpha_{c}(a)=\{x \in \alpha(\alpha):\|x\|=\|a\| \text { and }\|x\| \leq\|y\| \text { for } y \in \alpha(a)\} .
$$

In general, $\alpha_{c}(a)$ may be empty. In order to have an order theoretic characterization for $\alpha_{c}(\alpha) \neq \varnothing$, we need the following definition. The norm of an orthogonally decomposable space $B$ is said to be orthogonally monotone if the following condition is satisfied: if $x \geq \pm a$, and $x+a$ and $x-a$ are orthogonal, then $\|x\| \geq\|a\|$. Obviously, absolutely monotone norms are orthogonally monotone.

(2.2). The following two conditions are equivalent:

(1) $\alpha_{c}(a) \neq \emptyset$ for every $a \in B$;

(2) (i) the norm of $B$ is orthogonally monotone, (ii) every element $a \in B$ admits an orthogonal decomposition $a=b-c$ such that $\|a\|=\|b+c\|$.

Proof. (1) $\Rightarrow(2)$. To prove (i), suppose that $x \geq \pm a$, and $x+a$ and $x-a$ are orthogonal. It then follows from (2.1) that $x \in \alpha(a)$. Hence, by the assumption, $\|x\| \geq\|a\|$. To prove (ii), we take an arbitrary element $x \in \alpha(a)$, and set $b=\frac{1}{2}(x+a)$ and $c=\frac{1}{2}(x-a)$. Then $a=b-c$ is the required decomposition.

(2) $\Rightarrow(1)$. Let $a=b-c$ be the orthogonal decomposition determined by (2) (ii). Then, for $x=b+c$, we have $\|x\|=\|a\|$. Furthermore, for any $y \in \alpha(a)$, since $y \geq \pm a$, and $\frac{1}{2}(y+a)$ and $\frac{1}{2}(y-a)$ are orthogonal by (2.1), we have $\|y\| \geq\|a\|$ by (2) (i). Thus $x \in \alpha_{c}(a)$. 
It is clear that $\alpha_{c}(a) \neq \emptyset$ for every $a \in B$ if $B$ is uniquely orthogonally decomposable or a Banach lattice or the hermitian part of a $C^{*}$-algebra.

When $B$ is orthogonally decomposable, the dual $B^{*}$ is also orthogonally decomposable by (5.2) in [10]. Therefore the absolute value $\alpha(f)$ is also defined in $B^{*}$.

(2.3). $\alpha_{c}(F(a)) \subset H(x)$ for every $x \in \alpha_{c}(\alpha)$.

Proof. For $f \in F(a)$ and $x \in \alpha_{c}(a)$, we have

$$
\|x\|=\|a\|=\|f\|=\|g\|
$$

and

$$
\|x\|^{2}=\|a\|^{2}=(f, a) \leq(g, a) \leq\|g\| \cdot\|x\|=\|x\|^{2}
$$

for every $g \in \alpha_{c}(f)$. Hence $\alpha_{c}(f) \subset H(x)$.

REMARK. Let $B=C[-1,1]$, with the pointwise order, as in Example 4 after (3.2) in [11]. Let $a(t)=t$ for $t \in[-1,1]$. Then, if

$$
x(t)=\left\{\begin{array}{lll}
t+2 & \text { for } & -1 \leq t \leq 0, \\
-t+2 & \text { for } & 0 \leq t \leq 1,
\end{array}\right.
$$

we have $x \in \alpha(a)$. However, $\alpha(F(a))$ is not contained in $H(x)$.

It is not known whether the equality holds in (2.3) in general. When $B$ is a Hilbert space with a self-dual positive cone, it obviously does.

(2.4). Let $B$ be a o-complete Banach lattice. Then

$$
\alpha_{c}(F(a))=H(|a|) \text { for every } a \in B \text {. }
$$

Proof. Since $|a| \in \alpha_{c}(a), \alpha_{c}(F(a)) \subset H(|a|)$ follows from (2.3). Now let $f \in H(|a|)$ and set $g=f \circ P-f \circ(1-P)$, where $P$ is the projection defined by

$$
P x=\vee\left\{x \wedge n a^{+}: n=1,2, \ldots\right\} \text { for all } x \in B_{+} \text {. }
$$

Then $(g, a)=\left(f, a^{+}\right)+\left(f, a^{-}\right)=(f,|a|)=\|a\|^{2}=\|f\|^{2}$. This, in particular, implies $\|g\| \geq\|f\|$. On the other hand, since $g \leq f \circ P \leq f$ 
and $-g \leq f \circ(1-P) \leq f$, we have $\|g\| \leq\|f\|$. Therefore $\|g\|=\|f\|=\|a\|$ and, hence, $g \in F(a)$. Furthermore, for $x \in B_{+}$,

$$
\begin{aligned}
\left(g^{+}, x\right) & =\sup \{(g, y): 0 \leq y \leq x\} \\
& \geq(g, P x)=(f \circ P, x) .
\end{aligned}
$$

This shows that $g^{+} \geq f \circ P$. However, it follows from $g \leq f \circ P$ and $f \circ P \geq 0$ that $g^{+} \leq f \circ P$. Therefore $g^{+}=f \circ P$. Similarly $g^{-}=f \circ(1-P)$. Thus $|g|=f$. This shows that $f \in \alpha_{c}(F(a))$.

\section{The orthogonally decomposable homomorphisms}

Let $B$ be an orthogonally decomposable space. A continuous linear map $\phi: B \rightarrow B$ is called an orthogonally decomposable homomorphism if, for every $a \in B$, there exists an orthogonal decomposition $a=b-c$ such that $\phi(a)=\phi(b)-\phi(c)$ is also an orthogonal decomposition. When $\phi$ is an orthogonally decomposable homomorphism, such an orthogonal decomposition is called a proper decomposition for $\phi$. If every orthogonal decomposition of $a$ is proper for $\phi$ for every $a \in B$, $\phi$ is called a strong orthogonally decomposable homomorphism.

The following statement follows immediately from (2.1).

(3.1). Let $B$ be an orthogonally decomposable space and $\phi$ be a continuous linear map on $B$. The following conditions are equivalent:

(1) $\phi$ is an orthogonally decomposable homomorphism;

(2) for every $a \in B$, there exists $x \in \alpha(a)$ such that $\phi(x) \in \alpha(\phi(a))$.

The following conditions are equivalent:

(3) \$ is a strong orthogonally decomposable homomorphism;

(4) $\phi(\alpha(a)) \subset \alpha(\phi(a))$ for every $a \in B$.

When $B$ is a Banach lattice, every lattice homomorphism is an orthogonally decomposable homomorphism. When $B$ is the hermitian part of a $C^{*}$-algebra, the restriction of every Jordan homomorphism to $B$ is an orthogonally decomposable homomorphism. In this case, see [5] for a result similar to (3.1). It is clear that, if $B$ is uniquely orthogonally decomposable, every orthogonally decomposable homomorphism is strong. This 
is the case when $B$ is a Hilbert space with a self-dual positive cone.

The following statement has been proved in [11], (4.1), where a (strong) orthogonally decomposable automorphism is a bijection $\phi$ such that $\phi$ and $\phi^{-1}$ are both (strong) orthogonally decomposable homomorphisms.

(3.2). Let $B$ be an orthogonalzy decomposable space and $\phi: B \rightarrow B$ be a continuous linear bijection. The following conditions are equivalent:

(1) $\phi$ is a strong orthogonally decomposable automorphism and $\|\phi(x)\|=\|x\|$ for alz $x \in B_{+}$;

(2) $N(\phi(x))=N(x)$ for all $x \in B$;

(3) $\phi^{*} H(\phi(x))=H(x)$ for alz $x \in B$.

One consequence of this statement is the following:

(3.3). Let $B$ be an order unit space with the unit 1 and $\phi: B \rightarrow B$ be a continuous linear bijection such that $\phi\left(B_{+}\right)=B_{+}$and $\phi(1)=1$. Then $\phi$ is a strong orthogonally decomposable automorphism and $\|\phi(x)\|=\|x\|$ for alz $x \in B_{+}$.

Proof. We shall use condition (2) in (3.2). First we observe that $\phi^{*}\left(B_{+}^{*}\right)=B_{+}^{*}$, and $\left\|\phi^{*}(f)\right\|=\|f\|$ for all $f \in B_{+}^{*}$, because $\left\|\phi^{*} f\right\|=\left(\phi^{*} f, I\right)=(f, I)=\|f\|$ for all $f \in B_{+}^{*}$. Hence, for every $x \in B$, we have

$$
\begin{aligned}
N(\phi(x)) & =\sup \left\{(f, \phi(x)): f \in B_{+}^{*},\|f\| \leq 1\right\} \\
& =\sup \left\{\left(\phi^{*}(f), x\right): f \in B_{+}^{*},\|f\| \leq 1\right\} \\
& =\sup \left\{(g, x): g \in B_{+}^{*},\|g\| \leq 1\right\}=N(x) .
\end{aligned}
$$

Let us recall that a continuous linear bijection $\phi: B \rightarrow B$ is called an order automorphism if $\phi\left(B_{+}\right)=B_{+}$. When $B$ is a Banach lattice, every order automorphism is a lattice automorphism and, hence, an orthogonally decomposable automorphism. The statement (3.3) shows that, when $B$ is the hermitian part of a $C^{*}$-algebra, every order automorphism $\phi$ such that $\phi(1)=I$ is a strong orthogonally decomposable automorphism. However, the situation is different when $B$ is a Hilbert space with a self-dual positive cone.

Let $M$ be a von Neumann algebra on a Hilbert space $H$ such that 
there exists a cyclic and separating vector $\xi_{0} \in H$ for $M$. We equip the Hilbert space with the order structure defined by the self-dual cone $H_{+}=P_{\xi_{0}}^{G}$. For the details concerning this order structure, see [4]. As in [4], we denote the modular conjugation by $J$. In the following statement, $H$ is a complex Hilbert space. However, all maps concerned are from the real space $H^{J}$ into itself, where $H^{J}=H_{+}-H_{+} \cdot$

(3.4). Let $M$ be a von Neumann algebra on a Hilbert space $H$ as above. The following conditions are equivalent:

(1) $M$ is abelian;

(2) every order automorphism is an orthogonally decomposable automorphism;

(3) for every a $\in M$, the operator aJaJ: $H \rightarrow H$ is an orthogonally decomposable homomorphism.

Proof. We only need to prove the implications $(2) \Rightarrow(1)$ and (3) $\Rightarrow(1)$.

$(2) \Rightarrow(1)$. Let $a \in M$ and $\delta=a+j(a)$, where $j(a)=J a J$. Then, by [4], Theorem 3.4, $\delta$ is a continuous linear operator on $H$ and $e^{t \delta}$ are order automorphisms of $H$ for every $t \in R$. Hence, by the assumption,

$$
\left(e^{t \delta} \xi, e^{t \delta_{n}} \eta\right)=0 \text { if } \xi \in H_{+}, n \in H_{+} \text {and }(\xi, n)=0 .
$$

Since this is true for every $t \in R$ and

$$
(\delta \xi, \eta)=0 \text { if } \xi \in H_{+}, \eta \in H_{+} \text {and }(\xi, \eta)=0
$$

by [4], Lemma 5.3, we can conclude that

$$
\left(\delta^{2} \xi, \eta\right)+2(\delta \xi, \delta \eta)+\left(\xi, \delta^{2} n\right)=0
$$

for such $\xi$ and $\eta$. Now suppose that $a=a^{*}$. Then $\delta=\delta^{*}$ and it follows from the above equality that

$$
(\delta \xi, \delta \eta)=0 \text { if } \xi \in H_{+}, \quad \eta \in H_{+} \text {and }(\xi, \eta)=0 .
$$

Next, take projections $p$ and $q$ in $M$ such that $p q=0$, and set $\xi=p j(p) \xi_{0}$ and $\eta=q j(q) \xi_{0}$. Then $\xi \in H_{+}, \eta \in H_{+}$and $(\xi, n)=0$. 
Hence

$$
\begin{aligned}
0 & =\left((a+j(a)) p j(p) \xi_{0},(a+j(a)) q j(q) \xi_{0}\right) \\
& =2\left(q a p j(q a p) \xi_{0}, \xi_{0}\right) .
\end{aligned}
$$

Therefore $p q p=0$. When we consider the case where $q=z(p)-p$ for the central support $z(p)$ of $p$, we arrive at $a p=p a p$ and hence, $a p=p a$. Therefore every projection in $M$ is central. This means that $M$ is abelian.

(3) $\Rightarrow(1)$. Let $a \in M$ and $p$ be an arbitrary projection in $M$. Then, for $q=z(p)-p$, we have

$$
0=\left(a j(\alpha) p j(p) \xi_{0}, a j(a) q j(q) \xi_{0}\right)=\left(q a^{*} a p j\left(q a^{*} a p\right) \xi_{0}, \xi_{0}\right) \text {. }
$$

The rest of the proof is the same as above.

REMARK. We have actually proved that $a^{*} \alpha \in M \cap M^{\prime}$ if aJaJ is an orthogonally decomposable homomorphism.

\section{Quasi-domains of generators}

Let $B$ be an orthogonally decomposable space. In this section we shall only consider the linear operators $G: B \rightarrow B$ that are the generators of some $C_{0}$-semigroup $\left\{S_{t}: t \geq 0\right\}$ of continuous linear operators on $B$. In general, it is too restrictive to assume that the domain $D(G)$ of such $G$ satisfies $\alpha(\alpha) \subset D(G)$ whenever $a \in D(G)$. It is for this reason that we introduce the notion of quasi-domains of generators.

For every generator $G$ of a $C_{0}$-semigroup $\left\{S_{t}: t \geq 0\right\}$, we set

$$
(\bar{G}, a, f)_{+}=\underset{t \rightarrow 0+}{\lim \sup }\left(\left(s_{t} a-a\right) / t, f\right)
$$

and

$$
(G, a, f)_{+}=\underset{t \rightarrow 0+}{\lim \inf }\left(\left(S_{t} a-a\right) / t, f\right)
$$

for every $a \in B$ and $f \in B^{*}$. Obviously,

$$
(\bar{G}, a, f)_{+}=(\underline{G}, a, f)_{+}=(G a, f) \text { if } a \in D(G) \text {. }
$$

(4.1). Suppose that all $S_{t}$ are positive, that is, $S_{t}\left(B_{+}\right) \subset B_{+}$for 
all $t \geq 0$. Then $(\underline{G}, b, f)_{+} \geq(G a, f)$ for every $a \in D(G), b \in a_{+}$ and $f \in H(a)$.

Proof. Let $a=b-c$ be an orthogonal decomposition. Then it follows from $f \in H(a)$ that $(f, c)=0$, and

$$
\left(s_{t} a-a, f\right)=\left(s_{t} b-b-s_{t} c, f\right) \leq\left(s_{t} b-b, f\right),
$$

because $S_{t} c \in B_{+}$and $f \in B_{+}^{*}$.

The quasi-domain $Q(G)$ of a generator $G$ is defined by $Q(G)=\left\{a \in B:(\underline{G}, b, f)_{+}>-\infty\right.$ for every $b \in a_{+}$and $\left.f \in H(a)\right\}$.

Then it follows from (4.1) that $D(G) \subset Q(G)$ if all $S_{t}$ are positive. The converse is not true. In fact, it is known that, if all $S_{t}$ are contractions, every $S_{t}$ is positive if and only if $G$ is $N$-dissipative, that is, $(G a, f) \leq 0$ if $a \in D(G)$ and $f \in H(a)$ ([2], Theorem 4.1, or [3], Theorem 2.24).

(4.2). Let $G$ be the generator of a contraction semigroup $\left\{S_{t}: t \geq 0\right\}$. Then the following conditions are equivalent:

(1) $G$ is N-dissipative;

(2) $(\bar{G}, a, f)_{+} \leq 0$ for alz $a \in B$ and $f \in H(a)$.

Proof. $(1) \Rightarrow(2)$. Let $a \in B$ and $f \in H(a)$. Then, since $f \in B_{+}^{*}$ and $S_{t}\left(B_{+}\right) \subset B_{+}$,

$$
\left(s_{t} a, f\right) \leq\|f\| N\left(s_{t} a\right) \leq\|f\| \cdot\left\|s_{t}\right\| N(a) \leq N(a)^{2}=(a, f) .
$$

This implies $(\bar{G}, a, f)_{+} \leq 0$.

$(2) \Rightarrow(1)$. This follows immediately from $(\bar{G}, a, f)_{+}=(G a, f)$ for $a \in D(G)$.

Unlike the domain $D(G)$, the quasi-domain $Q(G)$ inherits some order structure from $B$.

(4.3). (i) If there exists $b \in a_{+}$such that $b \in Q(G)$, then $a \in Q(G)$.

(ii) If $B^{*}$ is strictly convex and $a \in Q(G)$, then $a_{+} \subset Q(G)$. 
Proof. (i) Suppose that $f \in H(a)$ and $a=b-c$ is an orthogonal decomposition such that $b \in Q(G)$. Then, since

$$
H(a)=F(b) \cap B_{+}^{*} \cap c^{0}=H(b) \cap c^{0} \subset H(b),
$$

we have $f \in H(b)$. Since $b_{+}=\{b\}$, we have $a \in Q(G)$.

(ii) When $B^{*}$ is strictly convex, $H(x)$ is a single-point set for every $x \in B$ (see [11], (H8)). Hence $H(a)=H(b)$ for every $b \in a_{+}$. Therefore $a \in Q(G)$ implies $b \in Q(G)$.

\section{A Kato inequality}

The Kato inequality [6] is a distributional inequality:

$$
\Delta|a| \geq(\operatorname{sgn} a) \Delta a
$$

for $a \in L_{\text {loc }}^{1}$ and $\Delta a \in L_{\text {Ioc }}^{1}$. In [8], there is a version of this, which can be stated as follows. Let $G$ be an operator on $L^{2}(M, d \mu)$. It is said to obey the Kato inequality if

(1) $a \in D_{f}(G)$ implies $|a| \in D_{f}(G)$,

(2) for $a \in D(G)$ and $f \in D_{f}(G)$ with $f \geq 0$,

$$
(f, G|a|) \geq((\operatorname{sgn} a) f, G a),
$$

where $D_{f}(G)$ is the form domain of $G$ and

$$
(\operatorname{sgn} a)(t)= \begin{cases}0 & \text { if } a(t)=0, \\ a(t) /|a(t)| & \text { otherwise. }\end{cases}
$$

Suppose that a linear operator $G$ is the generator of a $C_{0}$-semigroup $\left\{S_{t}\right\}$. It was proved in [8], Theorem 2.1, that, when $G$ is negative definite, $G$ obeys the Kato inequality if and only if all $S_{t}$ are positive.

A Banach space version of the Kato inequality and its relations to positive semigroups have been obtained in [1].

To establish a correspondence between the Kato inequality for $G$ and the positivity of $\left\{s_{t}\right\}$ in orthogonally decomposable spaces, we shall 
introduce another version of the Kato inequality.

For each $a \in B$, we set

$$
s(a)=\left\{f \in B_{+}^{*}: f(a)^{2}=f(x)^{2} \text { for all } x \in \alpha(a)\right\} .
$$

(5.1). (i) $s(a)=\left(b^{0} \cup c^{0}\right) \cap B_{+}^{*}$ for any orthogonal decomposition $a=b-c$.

(ii) $H( \pm a) \subset s(a)$.

Proof. (i) Let $f \in s(a)$ and $a=b-c$ be an orthogonal decomposition. Then, since $b+c \in \alpha(a)$, we have $f(b) f(c)=0$ and, hence, $f \in b^{0} \cup c^{0}$. To prove the converse, let $x \in \alpha(a)$. Then it follows from (2.1) and the assumption that $f(x)^{2}=f(a)^{2}$.

(ii) When $a=b-c$ is an orthogonal decomposition,

$$
H(a)=F(b) \cap B_{+}^{*} \cap c^{0} \text { and } H(-a)=F(c) \cap B_{+}^{*} \cap b^{0} .
$$

Hence $H(a) \cup H(-a) \subset\left(b^{0} \cup c^{0}\right) \cap B_{+}^{*}$.

A linear operator $G$ which is a generator of some $C_{0}$-semigroup is said to satisfy the Kato inequality if

(KI) $D(G) \subset Q(G)$,

(K2) $(\underline{G}, x, f)_{+}(x, f) \geq(G a, f)(a, f)$ for all $a \in D(G)$, $x \in \alpha(a)$ and $f \in s(a)$.

(5.2). Let $G$ be the generator of a $C_{0}$-semigroup $\left\{S_{t}\right\}$.

(i) If all $S_{t}$ are positive, $G$ satisfies the Kato inequality.

(ii) If $G$ is dissipative (that is, (Ga, $f) \leq 0$ for all a $\in D(G)$ and $f \in F(a)$ ) and satisfies the Kato inequality, then all $s_{t}$ are positive.

Proof. (i) The condition (KI) follows from (4.1). Now, let $a \in D(G), x \in \alpha(a)$ and $f \in s(a)$. Then

$$
\left|\left(s_{t} a, f\right)\right| \leq\left(s_{t} x, f\right) \text { and }|(a, f)| \leq(x, f) \text {. }
$$

Therefore 


$$
\begin{aligned}
\left(s_{t} a-a, f\right)(a, f) & =\left(s_{t} a, f\right)(a, f)-(a, f)^{2} \\
& \leq\left(s_{t} x, f\right)(x, f)-(x, f)^{2}=\left(s_{t} x-x, f\right)(x, f) .
\end{aligned}
$$

Therefore $G$ satisfies condition (K2).

(ii) Since $G$ is assumed to be dissipative, $\left\{S_{t}\right\}$ is a contraction semigroup. Hence we only need to prove that $G$ is $N$-dissipative. Let $a \in D(G)$ and $f \in H(a)$. Let $a=b-c$ be an orthogonal decomposition. Then, since $f \in F(b)$ and $\left\|S_{t}\right\| \leq 1$, we have $(\bar{G}, b, f)_{+} \leq 0$. Therefore, since $(\bar{G}, b, f)_{+}>-\infty$,

$$
(G a, f) \leq(\bar{G}, b, f)_{+}-(\underline{G}, c, f)_{+} \leq-(\underline{G}, c, f)_{+} \cdot
$$

on the other hand it follows from condition (K2) that

$$
(G a, f) \leq(\underline{G}, b+c, f)_{+} \leq(\bar{G}, b, f)_{+}+(\underline{G}, c, f)_{+} \leq(\underline{G}, c, f)_{+},
$$

because $b+c \in \alpha(a)$ and $f \in s(a)$. Thus we can conclude that $(G a, f) \leq 0$ and, hence, $G$ is $N$-dissipative.

\section{Locality of generators}

In [7], Nagel and Uhlig have proved that, when $B$ is a Banach lattice, every generator $G$ of a $C_{0}$-semigroup of lattice homomorphisms is local, that is,

$$
\text { Ga } \in a^{\underline{1} 1} \text { for every } a \in D(G)
$$

where $a^{\perp}=\{x \in B:|x| \wedge|a|=0\}$. A generalization of this fact, involving the second dual of $B$, has been obtained in [1].

When $B$ is $\sigma$-complete, the set $a^{\perp \perp}$ is equal to $P_{a}(B)$ for the projection $P_{a}$ defined by

$$
P_{a}^{x}=\bigvee_{n=1}^{\infty}(x \wedge n|a|) \text { for every } x \in B_{+} .
$$

Let $B$ be an ordered Banach space equipped with a closed and proper positive cone $B_{+}$. A hereditary subcone $F$ of $B_{+}$is called a face of $B_{+}$. When $F$ is a face of $B_{+}$, it has heen proved in [8], Theorem 8 , 
that $F^{N}$ is a closed face of $B_{+}$and

$$
F^{N}=\left\{x \in B_{+}:(f, x)=0 \text { if } f \in B_{+}^{*} \text { and }(f, y)=0 \text { for every } y \in F\right\},
$$

where $F^{N}$ is the $N$-closure of $F$ defined by

$$
F^{N}=\left\{x \in B_{+}: N\left(x-x_{n}\right) \rightarrow 0 \text { for some } x_{n} \in F\right\}
$$

and $N$ is the canonical half-norm associated with $B_{+}$. We shall denote ${ }_{F^{N}}$ by $C F$.

For $a \in B_{+}$, let $F_{a}$ be the face of $a$ in $B_{+}$, that is, $F_{a}$ is the smallest face in $B_{+}$containing $a$. Then

$$
F_{a}=\left\{x \in B_{+}: x \leq \alpha a \text { for some } \alpha \geq 0\right\} \text {. }
$$

Therefore

$$
C F_{a}=\left\{x \in B_{+}:(f, x)=0 \text { if } f \in B_{+}^{*} \text { and }(f, a)=0\right\} .
$$

When $B$ is a $\sigma$-complete Banach lattice, it has been proved in [8], Proposition 6, that $P_{a}\left(B_{+}\right)=C F$ for every $a \in B_{+}$, and, furthermore, $P_{a}(B)$ is the closed linear subspace spanned by $P_{a}\left(B_{+}\right)=C F F_{a}$. Moreover, we have $0 \leq P_{a} x \leq x$ for every $x \in B_{+}$.

Suppose that $H$ is a Hilbert space, $M$ is a von Neumann algebra on $H$ and there is a cyclic and separating vector $\xi_{0} \in H$ for $M$. As in $\S 2$, we consider the self-dual cone $H_{+}=P_{\xi_{0}}^{4}$ by which $H^{J}=H_{+}-H_{+}$is an ordered Hilbert space. Then, j.t has been proved in [4], Theorem 4.2, that for every $\xi \in H_{+}$there exists a projection $a \in M$ such that $a j(a) H_{+}=\bar{F}_{\xi}$, where $j(a)=J a J$. Furthermore, it is easy to see that $\bar{F}=C F_{\xi}$. If we denote $a j(a)$ by $P_{a}$, then $P_{a}$ is a projection operator on $H$ and $P_{a}(H)$ is the closed linear subspace spanned by $P_{\alpha}\left(H_{+}\right)=C F_{\xi}$. Moreover, $P_{a} \xi \in H_{+}$for every $\xi \in H_{+}$. However, unlike the case of Banach lattices, we do not always have $P_{a} \xi \leq \xi$ for $\xi \in H_{+}$.

(6.1). Let $M$ be a von Neumann algebra on a Hilbert space $H$ and 
suppose that there is a cyclic and separating vector $\xi_{0} \in H$ for $M$. Let $H_{+}=P_{\xi_{0}}^{4}$ and $a \in M$ be a projection. Then, for $P_{a}=a j(a)$, the following conditions are equivalent:

(1) $P_{a} \xi \leq \xi$ for every $\xi \in H_{+}$;

(2) a is a central projection.

Proof. (1) $\Rightarrow(2)$. Suppose that $\xi \in H_{+}, \eta \in H_{+}$and $(\xi, \eta)=0$. Then, since $H_{+}$is self-dual, $0 \leq\left(P_{a} \xi, P_{a} \eta\right) \leq(\xi, \eta)=0$. Hence $P_{a}$ is an orthogonally decomposable homomorphism. It then follows from (3.4) that $a$ is central.

$(2) \Rightarrow(1)$. If $a$ is central, it follows from [4], Proposition 4.10, that $1-P_{a}=(1-a) J(1-a) J$. Therefore $\left(1-P_{a}\right) \xi \in H_{+}$for every $\xi \in H_{+}$.

Returning to the definition of locality given by Nagel and Uhlig cited above, we shall try to find the corresponding form in general orthogonally decomposable spaces. Let $\left[\mathrm{CF}_{a}\right]$ be the closed linear subspace spanned by $C F_{a}$. We also define a closed linear subspace $[a]$ for every $a \in B_{+}$by

$$
[a]=\left\{x \in B:(f, x)=0 \text { if } f \in B_{+}^{*} \text { and }(f, a)=0\right\} .
$$

Then it is obvious that $[a] \cap B_{+}=C F_{a}$. Furthermore, when $B$ is a Banach lattice or the Hilbert space $H^{J}$ considered above, we have $\left[C F_{a}\right] \subset[a]$ and the inclusion is strict because $\left[C F_{a}\right]=[a]$ in the case of $H^{J}$ if and only if the cyclic projection $p_{a}$ associated with $a$ is central. Using these two subspaces $\left[C F_{\alpha}\right]$ and $[a]$, we can consider the following five interpretations of Nagel and Uhlig's definition in the case when $B$ is a orthogonally decomposable space:

(1) $G a \in[a]$ for every $a \in D(G) \cap B_{+}$;

(2) $G a \in\left[C F_{a}\right]$ for every $a \in D(G) \cap B_{+}$;

(3) $\alpha(G a) \subset[a]$ for every $a \in D(G) \cap B_{+}$;

(4) $\alpha(G a) \subset\left[C F{ }_{a}\right]$ for every $a \in D(G) \cap B_{+}$; 
(5) $(f, \alpha(G a))=\{0\}$ if $(f, \alpha(a))=\{0\}$ and $a \in D(G)$.

It is easy to see that, when $B$ is either a Banach lattice or $B=H^{J}$ considered above, $(i)$ is implied by $(i+1)$ for $i=1,2,3,4$. Now we shall show that the conditions (2), (3), (4) and (5) are not suitable forms of locality in general orthogonally decomposable spaces.

(6.2). Let $M$ be a von Neumann algebra on a Hilbert space $H$ and suppose that there is a cyclic and separating vector $\xi_{0} \in H$ for $M$. Let $H_{+}=P_{\xi_{0}}^{G}$. Assume that the following statement is valid: if $G$ is the generator of a uniformly continuous group of orthogonally decomposable homomorphisms on $H$, then $G \xi \in\left[C F_{\xi}\right]$ for all $\xi \in D(G) \cap H_{+}$. Then $M$ is abelian.

Proof. Let $a \in M$ be a projection and consider a continuous linear operator $\delta$ on $H$ defined by $\delta=i a+j(i a)$. Then $\delta^{*}=-\delta$. For each $t \in R, e^{t \delta}\left(H_{+}\right)=H_{+}$and

$$
\left(e^{t \delta_{\xi}}, e^{t \delta} \eta\right)=\left(e^{t \delta^{*}} e^{t \delta} \xi, \eta\right)=(\xi, \eta)
$$

Therefore $\left\{e^{t \delta}: t \in R\right\}$ is a uniformly continuous group of orthogonally decomposable homomorphisms on $H$ and $\delta$ is the generator. Hence, by the assumption, we have $\delta \xi \in\left[C F_{\xi}\right]$ for every $\xi \in H_{+}$. Note that

$$
C F_{\xi}=p_{\xi}^{j\left(p_{\xi}\right) H_{+}} \text {and }\left[C F_{\xi}\right]=p_{\xi}^{j\left(p_{\xi}\right) H} \text {, }
$$

where $p_{\xi}$ is the cyclic projection in $M$ defined by $p_{\xi}=\left[M^{\prime} \xi\right]$, the projection to the closed linear subspace spanned by $M^{\prime} \xi$. Now, for every projection $x \in M$, since $\xi_{x}=x j(x) \xi_{0} \in H_{+}$, we can consider the face ${ }^{F_{\xi}}$ and the projection $p_{x}=\left[M^{\prime} \xi_{x}\right]$. Then we have

$$
\delta x j(x) \xi_{0} \in\left[C F_{\xi_{x}}\right]=p_{x} j\left(p_{x}\right) H
$$

Furthermore, since

$$
p_{x}=\left[M^{\prime} x j(x) \xi_{0}\right] \leq\left[M^{\prime} x \xi_{0}\right]=x,
$$

we have 


$$
\delta x j(x) \xi_{0} \in x j(x) H \text { for every projection } x \in M
$$

Therefore $x j(x) \delta x j(x)=\delta x j(x)$. However

$$
\delta x j(x)=i(a x j(x)-x j(a x))
$$

and

$$
x j(x) \delta x j(x)=i(x a x j(x)-x j(x a x)) .
$$

Hence

$$
a x j(x)-x j(a x)=x a x j(x)-x j(x a x),
$$

or

$$
(a x-x a x) j(x)=x j(a x-x a x) .
$$

Multiplying $x$ from the left, we have $x j(a x-x a x)=0$. Hence

$$
(a x-x a x) j(a x-x a x)=(a-x a) x j(a x-x a x)=0 .
$$

This implies $a x=x a x$ and, therefore, $a x=x a$. It follows that $M$ is abelian.

As to the remaining condition (1), we immediately note that the generator of every $C_{0}$-semigroup $\left\{S_{t}: t \geq 0\right\}$ of self-adjoint orthogonally decomposable homomorphisms on a Hilbert space $H$, ordered by a self-dual positive cone $H_{+}$, satisfies this condition. In fact, if $\xi \in D(G) \cap H_{+}$, $n \in H_{+}$and $(\xi, n)=0$, we have

$$
t^{-1}\left(s_{t} \xi-\xi, n\right)=t^{-1}\left(s_{t} \xi, n\right)=t^{-1}\left(s_{t / 2} \xi, s_{t / 2} n\right)=0 \text {. }
$$

This fact corresponds to the result of Nagel and Uhlig cited in the heginning of this section.

When $G$ is the generator of a $C_{0}$-group, we have a stronger result.

(6.3). Let $B$ be an orthogonalzy decomposable space and $G$ be the generator of a $C_{0}$-group $\left\{S_{t}: t \in R\right\}$ of continuous linear operators on $B$.

(i) If all $S_{t}$ are positive, then $G$ satisfies condition (I).

(ii) Suppose that $\left\|S_{t}\right\|=1$ for all $t \in R$ and $\alpha(a) \subset D(G)$ whenever $a \in D(G)$. Then, if $G$ satisfies condition (1), all $S_{t}$ are 
positive.

Proof, (i) follows immediately from

$$
\cdot\left(s_{t} a-a, f\right)=\left(s_{t} a, f\right) \geq 0 \text { for every } t \in R
$$

when $f \in B_{+}^{*}, a \in D(G) \cap B_{+}$and $(f, a)=0$. To prove (ii), let $a \in D(G)$ and $f \in H(a)$. Let $a=b-c$ be an orthogonal decomposition; then, since $c \in B_{+}$and $(f, c)=0$, we have $(f, G a)=(f, G b) \leq 0$. because $f \in F(b), G$ is dissipative and, furthermore, $b \in D(G)$ and $c \in D(G)$ by the assumption that $\alpha(a) \subset D(G)$. Hence $G$ is $N$-dissipative. Similarly $-G$ is also $N$-dissipative. Therefore every $S_{t}$ is positive.

To avoid imposing the severe restriction that $\alpha(a) \subset D(G)$ for all $a \in D(G)$, we give the following definition. The generator $G$ of a $C_{0}-$ group $\left\{S_{t}: t \in R\right\}$ of continuous linear operators on $B$ is said to be Zocal if $D(G) \subset Q(G)$ and

$$
(\bar{G}, c, f)_{-} \leq 0 \text { and }(\underline{G}, c, f)_{+} \geq 0
$$

whenever $c \in B_{+}, f \in B_{+}^{*}$ and $(f, c)=0$, where

$$
(\vec{G}, c, f)_{-}=\lim _{t \rightarrow 0_{-}} t^{-1}\left(s_{t} c-c, f\right)
$$

for all $c \in B$ and $f \in B^{*} .(\underline{G}, c, f)_{-}$is defined similarly. It is obvious that a local generator satisfies condition (1).

(6.4). Let $B$ be an orthogonalzy decomposable space and $G$ be the generator of a $C_{0}$-group $\left\{S_{t}: t \in R\right\}$ of isometries on $B$. Then the following conditions are equivalent:

(I) $S_{t}\left(B_{+}\right)=B_{+}$for all $t \in R$;

(2) $\pm G$ satisfy the Kato inequality;

(3) $G$ is Zocal.

Proof. In view of (4.1) and (5.1), we only need to prove that (3) implies (1), or, equivalently, (3) implies that $\pm G$ are $N$-dissipative. Let $f \in H(a)$ and $a \in D(G)$, and let $a=b-c$ be an orthogonal decomposition. Since $D(G) \subset Q(G)$ and $\left\|S_{t}\right\|=1$, we have 


$$
-\infty<(\bar{G}, b, f)_{+} \leq 0 \text { and } 0 \leq(\underline{G}, b, f)_{-}<+\infty .
$$

Hence by the assumption,

$$
(G a, f) \leq(\bar{G}, b, f)_{+}-(\underline{G}, c, f)_{+} \leq-(\underline{G}, c, f)_{+} \leq 0
$$

and

$$
(G a, f) \geq(\underline{G}, b, f)_{-}-(\bar{G}, c, f)_{-} \geq-(\bar{G}, c, f)_{-} \geq 0 .
$$

Therefore $(f, G a)=0$ and $\pm G$ are $N$-dissipative.

\section{References}

[1] Wolfgang Arendt, "Kato's inequality and spectral decomposition for positive $C_{0}$-groups", Manuscripta Math. 40 (1982), 277-298.

[2] Wolfgang Arendt, Paul R. Chernoff and Tosio Kato, "A generalization of dissipativity and positive semigroups", J. Operator Theory 8 (1982), 167-180.

[3] Charles J.K. Batty and Derek W. Robinson, "Positive one-parameter semigroups on ordered Banach spaces", Acta Appl. Math. (to appear).

[4] Alain Connes, "Charactérisation des espaces vectoriels ordonnés sousjacents aux algèbres de von Neumann", Ann. Inst. Fourier (Grenoble) 24 (1974), 121-155.

[5] L. Terrell Gardner, "Linear maps of $C^{*}$-algebras preserving the absolute value", Proc. Amer. Math. Soc. 76 (1979), 293-307.

[6] T. Kato, "Schrodinger operators with singular potentials", Israel $J$. Math. 13 (1973), 135-148.

[7] Rainer Nagel and Heinrich Uhlig, "An abstract Kato inequality for generators of positive operator semigroups on Banach lattices", J. Operator Theory 6 (1981), 113-123.

[8] Derek W. Robinson and Sadayuki Yamamuro, "Hereditary cones, order ideals and half-norms", Pacific J. Math. 110 (1984), 335-343.

[9] Barry Simon, "An abstract Kato's inequality for generators of positivity preserving semigroups", Indiana Univ. Math. J. 26 (1977), 1967-1073. 
[10] Sadayuki Yamamuro, "On linear operators on ordered Banach spaces", Bull. Austral. Math. Soc. 27 (1983), 285-305.

[11] Sadayuki Yamamuro, "On orthogonally decomposable ordered Banach spaces", Bull. Austral. Math. Soc. 30 (1984), 357-380.

Department of Mathematics, Institute of Advanced Studies, Australian National University, GPO Box 4, Canberra, ACT 2601, Australia. 